Décadrages Décadrages

cinéma, à travers champs Cinéma, à travers champs

8-9| 2006

Le monde de Star Wars

\title{
L'empire vidéoludique : comment les jeux vidéo ont conquis l'univers de Star Wars
}

Dominic Arsenault et Bernard Perron

\section{(2) OpenEdition}

1 Journals

Édition électronique

URL : https://journals.openedition.org/decadrages/287

DOI : 10.4000/decadrages.287

ISSN : 2297-5977

Éditeur

Association Décadrages

Édition imprimée

Date de publication : 10 octobre 2006

Pagination : 98-105

ISBN : 978-2-9700582-4-3

ISSN : 2235-7823

Référence électronique

Dominic Arsenault et Bernard Perron, «L'empire vidéoludique : comment les jeux vidéo ont conquis l'univers de Star Wars », Décadrages [En ligne], 8-9 | 2006, mis en ligne le 04 février 2014, consulté le 03 avril 2022. URL : http://journals.openedition.org/decadrages/287 ; DOI : https://doi.org/10.4000/ decadrages. 287 


\title{
L'empire vidéoludique : comment les jeux vidéo ont conquis l'univers de Star Wars
}

\author{
par Dominic Arsenault et Bernard Perron
}

1 L'industrie du jeu vidéo a bien entendu auss flairé l'attrait du phénomène. Comme le note Steven L. Kent, les films Star Wars ont créé une certaine "vague d'enthousiasme pour la science-fiction (science-fiction mania)". Voir The Ultimate History of Video Games, Three Rivers Press, New York, 2001, p. 130.

2 Ce sont les frères Le Diberder qui, dès 1993 , donnent ce statut au jeu vidéo dans un titre de chapitre de Qui a peur des jeux vidéo? (La Découverte, Paris), ouvrage réédité en 1998 sous un autre titre: L'Univers des jeux vidéo (La Découverte, Paris).

3 Frank Beau, "Chronique d'une mutation annoncée", in Cahiers du cinéma, $n^{\circ} 503$, juin, 1996, p. 69.

4 Rusel Demaria et Johnny L. Wilson, "The Games Group: Early History of Games at LucasArts", in High Score! The Illustrated History of Electronic Games, Osborne, Berkeley, 2002, p. 198.

5 Ryder Windam, Star Wars. The Ultimate Visual Guide, DK Publishing, New York, 2005, p. 137.

6 Voir "Inside Lucasfilm. Company History", in Lucasfilm.com, en ligne à www.lucasfilm.com/ inside/ history $/$ ?page $=5$

\section{De Lucasfilm à LucasArts}

Il est difficile de discuter des développements intermédiaux de Star Wars sans évoquer la vision de l'instigateur de cet univers de science-fiction en constante expansion, George Lucas, qui a su mesurer l'importance qu'allait prendre le jeu vidéo (et, plus généralement, le phénomène des produits dérivés) ${ }^{1}$. Au-delà des considérations purement économiques, c'est d'abord par fascination personnelle que Lucas vient au $10^{\mathrm{e}} \mathrm{art}^{2}$. Dans une affirmation reprise dans les Cabiers du cinéma, il déclarait: "Le jeu vidéo, c'est la chose la plus excitante que j'aie découverte depuis que je suis dans le cinéma. C'est une combinaison de toutes les choses auxquelles je suis intéressé »3. Son entrée dans l'industrie du divertissement interactif est remarquée: en 1982, la célèbre société américaine Atari érige les fondations de l'empire vidéoludique de Lucas en investissant un million de dollars dans ce qui deviendra un département de Lucasfilm Ltd. consacré aux jeux vidéo 4 , première division de ce type à voir le jour dans une compagnie de production de films ${ }^{5}$.

Selon les informations données sur le site de la compagnie, Lucasfilm Games est créée "pour développer un prolongement interactif aux histoires et aux personnages" de Lucas ${ }^{\boldsymbol{6}}$. Les premiers jeux, Ballblazer (un jeu de soccer futuriste) en 1984 et Rescue on Fractalus! (un jeu de pilotage) en 1985 , ne répondent toutefois pas à cette orientation. Et si la division devient célèbre, c'est pour la parution de jeux d'aventures humoristiques tels que Maniac Mansion en 1987 et The Secret of Monkey Island en 1990 (Grim Fandango perpétuant toujours cette tradition en 1998). Bien que la série Indiana Jones connaisse sa première adaptation vidéoludique en 1989 avec Indiana Jones and the Last Crusade: The Graphic Adventure, il faut attendre encore quelques années avant que Lucasfilm Games n'exploite elle-même sa plus importante franchise. En effet, le premier jeu vidéo à paraître pour la console Atari 2600, Star Wars: The Empire Strikes Back en 1982, est développé par le grand fabricant de jeux de société Parker Brothers. 
Atari, quant à elle, introduit la guerre des étoiles dans les arcades en 1983 avec un jeu au titre homonyme. Après la parution de quelques titres entre 1983 et 1986, la franchise s'éclipse pendant plusieurs années. L'éditeur Brøderbund acquiert en 1988 les droits pour développer et publier des jeux vidéo basés sur Star Wars et autorise d'autres développeurs et éditeurs à les exploiter. Ce n'est qu'au moment où Lucasfilm Games devient une division de la LucasArts Entertainment Company que la société commence réellement à développer ses propres jeux avec Star Wars : $X$ Wing (1993). Selon toute évidence, la société a donc revisité sa propre histoire, puisque les sites officiels de Lucasfilm et de LucasArts imputent la fondation de cette dernière aux origines de la création des jeux:

«Depuis sa création en 1982, LucasArts a sans cesse été saluée pour sa création d'univers riches et immersifs que les joueurs sont amenés à découvrir. Les jeux de LucasArts sont continuellement applaudis par le critique et demeurent des succès commerciaux. Les produits de la compagnie raflent souvent les honneurs du ‘Jeu de l'année`, y compris de multiples récompenses de l'Academy of Interactive Arts ¿ Sciences. 7

7 Voir www.lucasarts.com/about/

Pourtant, répétons-le, la LucasArts Entertainment Company n'existe que depuis 1990.

\section{L'escadron vidéoludique}

La franchise de Star Wars compte à ce jour plus d'une centaine de jeux répartis selon plusieurs genres. Les jeux de tir et de pilotage se prêtent naturellement bien à cet univers. Des tout premiers jeux précédemment cités (1982 et 1983) à la série contemporaine Rogue Squadron (1998-2003) en passant par les deux Rebel Assault (1993 et 1995), le joueur est invité à prendre place dans le cockpit de différents vaisseaux et véhicules: on y trouve tout l'alphabet des -Wing (A, B, V, X, Y), les Speeders, ou le "Faucon Millénaire» de Han Solo. Si les objectifs premiers sont ici de détruire l'Etoile de la Mort ("Death Star») et d'abattre le plus de vaisseaux impériaux, une variante du jeu de pilotage consiste en des jeux de course dont les ambassadeurs sont Star Wars : Episode I-Racer (1999), Star Wars: Super Bombad Racing (2001) et Star Wars: Racer Revenge (2002).

L'autre genre populaire demeure le jeu de plateau traditionnel. Les plateformes de Nintendo ont accueilli plusieurs jeux basés sur les épisodes IV, V et VI. Star Wars (1991) et Star Wars: The Empire Strikes Back (1992), édités pour la Nintendo Entertainment System (NES), ont reçu un accueil très favorable:

En 1991, [la NES] donne à JVC et Lucasfilm l'opportunité de ressusciter la franchise vidéoludique de Star Wars. La NES était la première 
8 Skyler Miller, "The History of Star Wars Video Games. The Return of Games (1991-1998)", Gamespot.com, 2000, www.gamespot.com/ features/vgs/universal/swhistory/ret.html

9 L'hybridité des genres existe aussi en jeu vidéo et les jeux de la franchise ne font pas exception. Un jeu d'arcade comme Star Wars Trilogy (1998) mélange allègrement le jeu de tir, le jeu de pilotage et le jeu de combat à la première personne afin de recréer une expérience la plus proche de celle des films.

10 Au moment où nous écrivons ces lignes, la sortie officielle du jeu est annoncée avant la fin de l'année.

11 On entend par "troisième personne" les jeux où le joueur contrôle un avatar qu'il voit à l'écran. Quant aux jeux à la "première personne", il s'agit de jeux où le joueur contrôle le point de vue - une caméra subjective - d'un personnage qui n'est pas représenté à l'écran.

12 Les jeux massivement multijoueurs en ligne sont des jeux en temps réel auquel des centaines de milliers de joueurs du monde entier peuvent participer simultanément et dans lequel le monde virtuel évolue en permanence suivant les actions des joueurs connectés et les demandes formulées aux développeurs via la communauté branchée. console suffisamment puissante pour capturer l'imagerie, la sensation et l'histoire du film entier, plutôt que de se concentrer sur un seul aspect ou une seule scène 8 .

L'engouement pour la NES n'a pas encore tari que la Super Nintendo Entertainment System prend d'assaut la trilogie. Super Star Wars (1992), Super Empire Strikes Back (1993) et Super Return of the Jedi (1994) connaissent un vif succès. A l'exception de quelques passages où il pilote un vaisseau ${ }^{9}$, le joueur doit contrôler les mouvements de ses avatars de façon à vaincre différents obstacles et ennemis, et ainsi progresser à travers différents niveaux. Ce type de produit trouve un prolongement dans les jeux d'action en 3D comme Star Wars: Episode I- The Phantom Menace (1999) ou Star Wars Episode III: Revenge of the Sith (2005) qui, sortis en même temps que les films du même titre, s'attachent à mettre en scène les événements contés dans ceux-ci. Lego Star Wars: The Video Game (2005) et Lego Star Wars II: The Original Trilogy (2006) $\mathbf{1 0}$ proposent quant à eux une relecture plus légère des deux trilogies en reconstruisant tout l'univers en blocs Lego. Au lieu de diriger un avatar à la troisième personne $\mathbf{1 1}$ comme dans les titres cités précédemment, le joueur est aussi invité à contrôler son point de vue dans quelques jeux de tir à la première personne (FirstPerson Shooters ou FPS): Star Wars: Dark Forces (1995), Star Wars : Jedi Knight II - Jedi Outcast (2002) et Star Wars: Republic Commando (2005).

L'expansion territoriale de l'empire Star Wars a conduit à l'annexion des jeux de stratégie et des jeux de rôle. Inspirés des modèles de Warcraft et de Civilization, Star Wars: Force Commander (2000) et Star Wars: Empire at War (2006) proposent au joueur de diriger des troupes et de gérer des ressources. Avec Star Wars: Knights of the Old Republic I et II (2003 et 2004) et Star Wars Galaxies: An Empire Divided (2003), le joueur peut créer son propre personnage et vivre des aventures à la fois hors ligne et en ligne. D’ailleurs, l'univers de ce dernier jeu en ligne massivement multijoueurs $\mathbf{1 2}$ ne cesse de se complexifier à travers des modules d'extension, tels que Star Wars Galaxies: Jump to Ligh Speed (2003) et Star Wars Galaxies : Trials of Obi-Wan (2005).

Comme le démontre cette revue des troupes, le joueur est en mesure de s'enrôler dans la guerre des étoiles quel que soit le type de jeu qu'il préfère. S'il est impossible d'embrasser dans l'ensemble toute l'armada, on peut tout de même souligner quelles sont les stratégies de jeu (ou de guerre) les plus saillantes.

\section{Au-delà de l'univers filmique : naviguer dans l'hyper-espace}

Plusieurs observateurs sont portés à reconsidérer Un nouvel espoir (1977) à la lumière de l'importance qu'ont acquise les jeux vidéo. Aussi la per- 
ception rétrospective de Celia Pearce est-elle certainement partagée par plusieurs commentateurs: "Star Wars est un bon exemple de monde narratif fait sur mesure pour un jeu. Le premier film est bien plus un jeu qu'un film»13. Naturellement, l'univers transmédiatique de Star Wars se prête bien aux adaptations vidéoludiques, et ce pour plusieurs raisons.

Le désir de se retrouver dans la peau de personnages - les productions vidéoludiques Star Wars permettent par exemple de se mettre à la place de Luke Skywalker, de Darth Vader, de Han Solo, de la princesse Leia et même de Chewbacca ou d'un Ewok - auquel la littérature et le cinéma répondent à travers la fameuse notion d'"identification" aux personnages de la fiction (ou d'«empathie» avec eux) est pleinement satisfait dans les jeux vidéo. Au-delà de la question du genre, la majorité des œuvres du $10^{\mathrm{e}}$ art sont considérées comme des jeux de rôle. Une grande part du plaisir ludique n'est plus seulement de vibrer au rythme des péripéties vécues par les personnages, mais d'entrer réellement en phase avec les actions qu'ils effectuent. L'attrait du faire-semblant ne tient plus au regarder-faire, mais au faire-soi-même: au lieu de regarder Mark Hamill jouer un rôle, nous sommes invités à revêtir ses habits. Dans cette perspective, le choix et le statut des avatars que le joueur peut personnifier revêtent une grande importance. Car les aventures ou les quêtes sont encore plus extraordinaires lorsqu'il s'agit d'incarner des personnages hors du commun tels que le Jedi Luke Skywalker14 ou de devenir soi-même un utilisateur de la Force ou un pilote de la Rébellion. Qui plus est, comme dans les romans ou les bandes dessinées, l'univers vidéoludique permet de se concentrer sur certains personnages à peine croisés dans les films, et d'ajouter de nouveaux noms à la liste des acteurs de la saga. D’une part, et c'est peut-être l'exemple le plus connu, contrairement à sa figuration mineure sur le grand écran, le pilote Wedge Antilles devient un acteur de premier plan dans les batailles de la série Rogue Squadron. Un personnage comme Jango Fett, père du mythique Boba Fett, se voit consacré par l'entremise d'un jeu qui lui est dédié : Star Wars: Bounty Hunter (2002). Les dernières rumeurs annoncent même le développement par LucasArts d'un jeu destiné à présenter des pans jusqu'ici inconnus de l'histoire de Darth Vader15. D'autre part, et pour donner un dernier exemple, le désopilant drö̈de-assassin protocolaire de Star Wars: Knights of the Old Republic, HK-47, a fait une entrée remarquée dans le groupe des célébrités en remportant en 2004 le prix du meilleur personnage original au Game Developers Choice Awards $\mathbf{1 6}$.

L'envie de piloter un X-Wing ou de manier un sabre laser que peuvent nourrir certains spectateurs des films est exaucée à satiété dans l'univers virtuel. S'il était impossible d'affronter soi-même l'Empire galactique
13 Celia Pearce, "Towards a Game Theory of Game ", in Noah Wardrip-Fruin et Pat Harrigan (éd.), First Person. New Media as Story, Performance and Game, The MIT Press, Cambridge, MA, 2004, p. 153.

14 C'est toutefois dans la série de jeux vidéo Wing Commander que Mark Hamill, alias Luke, tiendra le premier rôle. II incarne le colonel Christopher Blair dans Wing Commander III: Heart of the Tiger (1994), Wing Commander IV: The Price of Freedom (1995) et Wing Commander: Prophecy (1997).

15 Laura M. Holson, "At LucasArts, No Playing Around in the Quest to Be No. 1", The New York Times, 17 avril 2006.

16 www.gamechoiceawards.com/archive/gdca _4th.htm 
17 Janet H. Murray, Hamlet on the Holodeck. The Future of Narrative in Cyberspace,The Free Press, New York, 1997, p. 126.

18 Skyler Miller, op. cit.

19 Henry Jenkins, "Game Design as Narrative Architecture ", in Noah Wardrip-Fruin et Pat Harrigan (éd.), op. cit., pp. 118-130. assis dans son siège de cinéma, le jeu vidéo, art de la simulation par excellence, nous offre toutes les possibilités de reproduire les actions cultes accomplies sur le grand écran et, littéralement, d'entrer dans le jeu. Tel que Janet Murray l'a explicité, les environnements virtuels interactifs nous confèrent le pouvoir de réaliser des actions significatives et d'en voir les résultats (ce qu'elle appelle l'agency) ${ }^{\mathbf{1 7}}$. Ainsi, des premiers vaisseaux conçus à partir de graphismes vectoriels dans le jeu d'arcade de 1983 aux modèles 3D extrêmement détaillés d'aujourd'hui, la joie de détruire ces derniers demeure tout aussi exaltante. Dès les premiers combats au sabre laser dans les jeux de plateau en 2D, le maniement de l'arme fétiche des Jedi était électrisant. L'apport du vrombissement caractéristique du sabre dans les nombreux affrontements d'un jeu comme Star Wars Episode III: Revenge of the Sith lui confère un effet plus authentique. Dans cette perspective, il est intéressant de signaler l'impact qu'a toujours eu le son sur le degré de fidélité de la reproduction. Analysant les réussites et les échecs du premier jeu d'arcade, Skyler Miller affirme:

"C'est l'utilisation de voix et d'effets sonores tirés du film qui rend le jeu vraiment complet. Et il ne s'agit pas de voix synthétisées par ordinateur à la Bezerk. Entendre Obi-Wan dire ‘Luke, utilise la Force〉 ou Han Solo crier ‘La route est dégagée p'tit gars!) ajoute cette dernière touche de réalisme requise pour propulser ce jeu de Star Wars au-delà du 〈bon jeu〉 et d'en faire un 〈grand jeu 〉.»18

Cette touche de réalisme nous conduit à la seconde caractéristique des environnements virtuels, à savoir la question de l'immersion.

Qui n'a pas eu envie d'explorer les méandres de la galaxie? C'est certes là l'une des grandes forces de Lucas et de sa création. Cette dernière est si extensive qu'en plus des multiples planètes à visiter, même l'estomac d'une limace géante de l'espace (Space slug) devient un lieu à découvrir. L'attrait des jeux vidéo tient évidemment à la possibilité de s'immerger complètement dans l'univers de Star Wars, reléguant ainsi, pendant la durée du séjour, notre monde réel au second plan. Le joueur peut non seulement se rendre sur Tatooine, Hoth ou Dagobah, mais aussi fouler le sol de destinations à peine évoquées à l'écran, telles que Dantooine (Knights of the Old Republic I et II), Kashyyyk (Star Wars: Republic Commando, 2005) ou la base Echo sur Hoth (Star Wars: Shadows of the Empire, 1996). L'immensité spatiale, à la fois géographique et intersidérale, est plus que propice à une exploitation vidéoludique puisque la question de l'espace est au cœur de la spécificité de ce médium.

Suivant Henry Jenkins, le design des jeux se conçoit en termes de construction d'espaces, et propose par ce fait même des architectures narratives 19. L'espace devient le vecteur principal à travers lequel sont 
véhiculées les histoires et les expériences du joueur. Ce dernier doit explorer l'environnement afin de vivre l'aventure ou de participer aux événements diégétiques. C'est pourquoi l'unique course de pods de l'Episode I peut faire l'objet d'un jeu entier (Star Wars Episode I: Racer). De même, alors que la bagarre à la cantine de Mos Eisley dans l'épisode IV se résume en fin de compte à une petite bousculade et à un seul coup de sabre laser d'Obi-Wan, ce lieu justifie à lui seul tout un niveau dans le jeu de plateau Super Star Wars. Non seulement Luke (ou même Chewbacca, si le joueur décide de l'incarner) devra cette fois-ci se battre contre des hordes hostiles $\mathbf{2 0}$ en avançant horizontalement (le jeu étant de type side-scrolling) dans cette cantine qui n'en finit plus, mais il lui faudra terrasser pour finir le gigantesque «Kalhar» afin de pouvoir quitter les lieux. Cette dilatation de l'action à travers celle de l'espace, un procédé somme toute classique quand vient le temps d'adapter un film en jeu, peut entraîner des transformations plus radicales. Si après avoir repéré la chute de ce qui ressemble à un météore dans l'épisode $\mathrm{V}$, Luke se fait attaquer par un "Wampa» qui le ramène inconscient à la maison avec l'intention d'en faire un succulent repas, ce tête-à-tête impromptu se déroule tout autrement dans Super Empire Strikes Back. En effet, avant même de sortir ses lunettes d'approche, Luke doit d'abord trouver sa monture (un "Tauntaun") en se frayant un chemin à travers un flot de créatures agressives et une avalanche de boules de neige géantes. Ce premier niveau terminé, le joueur devra encore traverser des steppes enneigées et un ensemble de cavernes dans lesquelles plusieurs "Wampas» ont élu domicile. L'attaque sournoise du yéti du film est remplacée par plusieurs face-à-face ludiques plus loyaux, qui culminent en un affrontement final contre un "Wampa" géant créé de toutes pièces par LucasArts pour les besoins de la cause 21 .

\section{Les deux côtés de la Force: Knights of the Old Republic}

Sachant que, sur le grand écran, la "guerre des étoiles» est désormais terminée, force est de constater qu'il faut se tourner vers les autres médiums pour jouir de nouveaux épisodes. Dans cette perspective, les jeux vidéo sont plus qu'en mesure de perpétuer à la fois le spectacle audiovisuel incomparable de Star Wars et la richesse de son univers fictionnel. Qui plus est, de simples versions annexées à la galaxie des deux trilogies, les jeux vidéo ont lentement acquis leur indépendance par rapport aux films. On retrouve les bases de cette autonomie dans Star Wars: TIE Fighter où, dès 1994, le joueur pouvait incarner grâce à l'interactivité un pilote impérial et affronter l'Alliance rebelle aux commandes de différents types de chasseurs (TIE Fighter, Bomber, Advanced,
20 II s'agit en l'occurrence d'espèces connues dans l'univers Star Wars: on y combat des Rodians, des Duros et des Shistavanens.

21 Le boss fight est une figure emblématique du jeu vidéo. II consiste en un combat final qui clôt un niveau ou un chapitre. Le joueur doit y vaincre un adversaire surpuissant, souvent une version de taille plus imposante que ses congénères rencontrés tout au long du niveau. 
22 Tous les prix sont énumérés sur la page du site officiel du jeu de la compagnie Bioware: www.bioware.com/games/knights_old_republic/ awards/

23 www.gamespot.com/xbox/rpg/starwars knightsoftor/review.html ?page $=3 \& q=\& q=$

24 Sélection d'une classe (soldat, aventurier ou canaille), distribution de points dans différents attributs (force, dextérité, etc.), détermination de compétences (discrétion, informatique, etc.) et de dons (entre autres, prudence et endurance), choix d'un sexe, d'un visage, et, finalement, d'un nom.
Defender, etc.). Un tel changement d'allégeance introduisait la possibilité de complexifier la dynamique manichéenne inscrite au cœur de ce conflit originel.

Dix ans plus tard (ou, dans la chronologie de Star Wars, 4000 ans avant la Bataille de Yavin), le jeu Knights of the Old Republic a parachevé cette relativisation de la logique binaire des films tout en devenant un jalon important à la fois de la franchise et du jeu vidéo (raflant plus de 125 prix 22). Comme le note Greg Kasavin dans sa critique disponible sur le site reconnu «Gamespot.com»:

"C'est l'un des seuls jeux de Star Wars qui fait réellement sentir au joueur qu'il est la clé de voûte de cet univers de science-fiction si unique et apprécié. [...] Il présente aussi une version de Star Wars beaucoup plus complexe moralement que celle vue dans les films. »23

"Choose your path». Cette promesse du slogan publicitaire de Knights of the Old Republic est tenue. Le joueur incarne un personnage qu'il a d'abord créé en fonction de paramètres propres aux jeux de rôle $\mathbf{2 4}$. Ce dernier sera ensuite impliqué dans une série d'événements le menant devant le Conseil des Jedi, qui fera de lui un des leurs. Avec un sabre laser et le pouvoir de la Force à sa disposition, le joueur doit visiter des planètes familières autant qu'inconnues en quête d'une carte menant à la Forge Stellaire (Star Forge). Mais le seigneur Sith Darth Malak est lui aussi sur la piste de ce mystérieux artefact extra-terrestre au pouvoir incommensurable afin de l'utiliser pour mener à terme son plan de domination galactique. Bien que les grandes lignes de l'intrigue soient déjà scénarisées, les détails ouvrent sur de multiples possibilités. Tout au long de son aventure, le joueur prend des décisions qui le feront pencher soit du côté lumineux de la Force, soit du côté obscur. Par exemple, sur Tatooine, lorsqu'il apprend que les gens vivant à Anchorhead sont attaqués régulièrement par des hommes des sables (Tusken Raiders), il peut décider de les éliminer sans pourparlers ou plutôt de négocier une trêve. Par son choix, il détermine si son personnage se range du côté obscur ou du côté lumineux de la Force. Mais les choses ne sont pas toujours aussi bien tranchées. Le conflit central opposant Darth Malak au Conseil Jedi est enraciné dans une lutte politique appelée les Guerres Mandaloriennes (Mandalorian Wars). Vivant en périphérie de la Bordure Extérieure (Outer Rim), les Mandaloriens ont commencé depuis quelques années à conquérir des planètes de la République. Les Jedi n'ont pas réagi à ces attaques. Sentant qu'une menace encore plus grande allait s'abattre sur eux s'ils se portaient à la rescousse de ces mondes, ils ont jugé plus sage de sacrifier un nombre incalculable de civils. Des 
Jedi dissidents comme Malak, ne pouvant rester les bras croisés, partirent en guerre pour mettre un terme à cette barbarie, mais passèrent du côté obscur de la Force en mettant les Mandaloriens en échec. Dans cette perspective, lorsque le joueur doit appuyer ou non les décisions du Conseil (entre autres, lorsqu'on lui demande de prendre position par l'entremise de dialogues au sommet du temple sur Rakata Prime), il fait face à une impasse. S'il soutient le Conseil Jedi, il approuve leur inaction passée dont les conséquences furent meurtrières. Mais il ne peut s’y opposer totalement, puisqu'en volant à la rescousse des innocents, les Jedi dissidents ont basculé du côté obscur et sont devenus la nouvelle menace. On comprend donc le commentaire de Kasavin. Il ne s'agit plus seulement de choisir entre le bien et le mal, entre le côté lumineux et le côté obscur de la Force; la nature interactive du jeu vidéo permet de placer le joueur devant de nouveaux dilemmes.

L'évolution historique de l'empire vidéoludique de LucasArts ainsi que nos commentaires sur des jeux récents laissent présager que ce type de productions jusqu'ici développées parallèlement aux films deviendra la pierre angulaire de la franchise. Avec un jeu en ligne massivement multijoueurs comme Star Wars: Galaxies où chaque joueur pour choisir sa destinée et influer sur le cours de l'Histoire, nul ne peut prédire ce qu'il adviendra de la galaxie lointaine, très lointaine ${ }^{25} \ldots$

25 Le slogan sur le site web officiel du jeu se lit d'ailleurs comme suit: "Where the movies left off, your adventure begins" (http://starwarsgalaxies.station.sony.com/). 